\title{
5 SATISFAÇÃO DE USUÁRIOS NUM CENTRO DE ATENÇÃO PSICOSSOCIAL EM ÁLCOOL E OUTRAS DROGAS
}

| Guilherme Barbosa'; Márcia de Oliveira²; Vânia Moreno³; Carlos Padovani'; Heloisa Claro5; Paula Pinho ${ }^{6}$ |

\section{RESUMO}

Este estudo de caráter descritivo, exploratório e transversal teve como objetivo avaliar a satisfação dos usuários de um Centro de Atenção Psicossocial em Álcool e Outras Drogas. Foram entrevistados 25 sujeitos, em um município do interior do Estado de São Paulo. Os dados foram coletados entre maio e julho de 2013. Utilizou-se a Escala de Satisfação dos Pacientes com os Serviços de Saúde Mental. A maioria dos usuários era do sexo masculino (68\%), com idade média de 43 anos, solteiros (48\%), com ensino fundamental incompleto (40\%), empregados (36\%), sendo que $76 \%$ possui renda própria. Os resultados apontaram que grande parte dos usuários estava satisfeito em relação aos aspectos: competência e compreensão da equipe sobre o problema do usuário, ajuda recebida e acolhida da equipe e no que se refere às condições físicas e conforto do CAPSad. Constatou-se a importância desse serviço aos usuários sendo fundamental para o tratamento dessa demanda.

PALAVRAS-CHAVE: Avaliação; Serviços de saúde mental; Saúde mental

\section{RESUMEN}

"Satisfacción de los usuarios en un Centro de Atención Psicosocial en alcohol y otras drogas"

Este estudio transversal descriptivo y exploratorio evalúa la satisfacción de los usuarios de un Centro de Atención Psicosocial de alcohol y otras drogas. Fueron entrevistados 25 sujetos en una ciudad en el interior del estado de São Paulo. Se recogieron datos de mayo hasta julio 2013 mediante la Escala de Satisfacción del Paciente con los Servicios de Salud Mental. La mayoría de los usuarios eran hombres (68\%) con una edad media de 43 años, solteros (48\%), con educación primaria incompleta (40\%), actualmente empleados (36\%), y tiene ingresos propios (76\%). Los resultados muestran que la mayoría de los usuarios estaban satisfechos con respecto a los siguientes aspectos: la competencia y la comprensión del equipo de salud acerca de los problemas de los usuarios; atención recibida; acogida por el equipo de salud; y en relación con las condiciones físicas y la comodidad de CAPSad. Se observó la importancia de este servicio a los usuarios, siendo fundamental para el tratamiento de esta demanda.

DESCRIPTORES: Evaluación; Servicios de salud mental; Salud mental

\section{ABSTRACT \\ "User satisfaction with Psychosocial Care Center for alcohol and other drugs"}

This cross-sectional descriptive and exploratory study aimed to evaluate the satisfaction of users of a Psychosocial Care Center on Alcohol and Other Drugs. 25 subjects were interviewed in a country side city in the state of São Paulo. Data were collected from May to July 2013 using the Patient Satisfaction Scale with Mental Health Services. Most users were male $(68 \%)$ with a mean age of 43 years, unmarried (48\%), with incomplete primary education (40\%), currently employed (36\%), 76\% have their own income. The results show that most users were satisfied regarding the following aspects: competence and understanding of the health team about the user's problems; care received; embracement by the health team; and regarding physical conditions and comfort of CAPSad. It was observed the importance of this service to users, being fundamental to the treatment of this demand.

KEYWORDS: Evaluation; Mental health services; Mental health

Submetido em 20-02-2015 - Aceite em 30-11-2015

\footnotetext{
1 Professor Doutor na Universidade Estadual Paulista, Faculdade de Medicina de Botucatu, Departamento de Enfermagem, Via Domingos Sartori, 18607-621 Botucatu - São Paulo, Brasil, gbarbosa@fmb.unesp.br

2 Professor Associado na Universidade de São Paulo, Escola de Enfermagem, Departamento de Enfermagem Psiquiátrica e Materno-Infantil, 05403-000 São Paulo, Brasil, marciaap@usp.br

3 Professor Doutor na Universidade Estadual Paulista, Faculdade de Medicina de Botucatu, Departamento de Enfermagem, São Paulo, Brasil, btmoreno@terra.com.br 4 Professor Titular no Instituto de Biociências de Botucatu, Departamento de Bioestatística, 18618-970 Botucatu - São Paulo, Brasil, padovani@ibb.unesp.br

5 Doutoranda do Programa de Pós-graduação em Enfermagem na Universidade de São Paulo, Escola de Enfermagem, 05403-000 São Paulo, Brasil, helo_gclaro@hotmail.com 6 Doutora pela Universidade de São Paulo, Escola de Enfermagem, 05403-000 São Paulo, Brasil, phpinho@terra.com.br
} 


\section{INTRODUÇÃO}

A atenção à saúde mental tem buscado a implantação de serviços substitutivos ao hospital psiquiátrico, com destaque aos Centros de Atenção Psicossocial (CAPS). Essa modalidade de atendimento foi implantada em diversos municípios brasileiros em substituição aos hospitais psiquiátricos, atendendo ao que é preconizado pela Reforma Psiquiátrica Brasileira. Os CAPS constituemse em um sistema comunitário de saúde mental e tem como uma de suas metas a inclusão social do indivíduo portador de transtorno mental (Amarante, 2008).

Neste contexto, os CAPS assumem relevância no cenário das novas práticas em saúde mental no país. Essa mudança paradigmática deve englobar a relação que se estabelece com o usuário, equipe e família, e entre esses e a comunidade. A mudança de papéis, a democratização das instituições, o envolvimento e responsabilização da comunidade devem somar-se ao tratamento. O cuidado na comunidade torna-se mais complexo, interdisciplinar exigindo que as práticas e saberes tradicionais sejam reconstruídos para responder à esta transformação (Amarante, 2008).

Com a Portaria 336/2002, se inicia a implantação dos Centros de Atenção Psicossoocial em Álcool e Outras Drogas (CAPSad), que passa a ser considerado um dos pilares para a construção de uma rede de serviços direcionada à população dependente de Álcool e Outras Drogas (AOD). De acordo com as propostas do Ministério da Saúde, estes dispositivos devem utilizar como referência a lógica da Redução de Danos, buscando atender às necessidades de seus usuários de forma sistemática e ativa, coadunando com o meio cultural e a comunidade em que estão inseridos, e de acordo com os princípios da Reforma Psiquiátrica (Brasil, 2003).

Com mais de uma década da implantação dos CAPSad, torna-se uma necessidade realizar processos avaliativos destes serviços. Estes processos devem monitorar as suas ações e acompanhar suas práticas de forma a construir um serviço que se proponha a atender a inserção do usuário, com maior resolubilidade, com uma intervenção pautada na diversidade de saberes de uma equipe multiprofissional e na utilização de recursos múltiplos.

Esta investigação objetivou avaliar o nível de satisfação dos usuários de um CAPSad, de um município no interior do estado de São Paulo.

\section{MÉTODO}

Trata-se de um estudo do tipo descritivo, exploratório e transversal. Consiste também de uma pesquisa avaliativa, do tipo somativa, que visa avaliar os resultados ou efeitos de serviços ou programas de tratamento (Furtado, 2001).

Foi realizado em um CAPSad de um município no interior do estado de São Paulo, em funcionamento desde 2007. Os dados foram coletados entre maio e julho de 2013. Entre os 30 usuários sorteados aleatoriamente, de um universo de 182 pacientes ativos, 25 participaram do estudo, dois não aceitaram, dois abandonaram e um estava internado em uma Comunidade Terapêutica no período da coleta.

O cálculo da amostragem foi realizado com base nos dados do estudo piloto. Os escores da escala de satisfação SATIS-BR variam de 1 a 5 . O desvio padrão mais alto dos domínios do questionário SATIS-BR foi estimado em 0,8 , valor este a partir do qual estimamos o tamanho adequado da amostra. Definimos a precisão da estimação com um intervalo de confiança de $95 \%$ em um erro padrão de 0,0215.

Assim, para a estimativa da média de satisfação da avaliação dos serviços dos CAPSad calculou-se uma amostra 30 usuários. A fórmula foi baseada em cálculo em que a margem de erro é representada como $Z{ }^{\star}$ Erro Padrão.

Dos 30 usuários sorteados, apenas 25 foram entrevistados.

Cabe ressaltar que este trabalho é um recorte do projeto de pesquisa "Avaliação dos Centros de Atenção Psicossocial em Álcool e Outras Drogas do Estado de São Paulo" conduzida pelo Grupo de Estudos de Álcool e outras Drogas da Escola de Enfermagem de São Paulo - GEADUSP.

Definiu-se como critérios de inclusão: ser maior de 18 anos de idade, estar cadastrado e participando das atividades dos CAPSad há no mínimo seis meses e ser portador de transtorno mental grave e persistente, decorrentes do uso e da dependência ao álcool e outras drogas. Utilizou-se como instrumento para a coleta de dados um questionário contendo as seguintes variáveis: sexo, idade, escolaridade, estado civil, renda própria, renda familiar, com quem vive (mora), idade em que percebeu o uso abusivo/dependência, internação psiquiátrica e uso de psicofármacos. 
Para avaliar a satisfação dos usuários com o serviço, utilizou-se a Escala de Avaliação da Satisfação dos Pacientes com os Serviços de Saúde Mental (SATIS-BR). Esta escala foi elaborada pela divisão de Saúde Mental da Organização Mundial de Saúde (WHO, 1996) e validada para o Brasil (Bandeira, Pitta, \& Mercier, 2000). Estudo recente de validação da escala aponta que a mesma possui índice de consistência interna (alfa de Cronbach) igual a 0,88 , o que é considerado válido pela literatura. Além disso, a escala apresentou validade convergente evidenciada pela comparação da correlação com escores de escala que mensura constructo semelhante.

A análise de correlação de cada fator com a escala global demonstrou correlação positiva e estatisticamente significante (Bandeira e Silva, 2012). SATIS-BR possui 12 itens, agrupados em três fatores para avaliar o grau de satisfação dos pacientes com relação à: Competência e compreensão da equipe a respeito do problema do paciente (Fator 1), Ajuda recebida e acolhida da equipe (Fator 2) e Condições físicas e conforto do serviço (Fator 3), incluídos também na escala global. As alternativas de resposta aos itens estão dispostas em uma escala do tipo Likert com 5 pontos, na qual 1 representa que o usuário está muito insatisfeito e 5 que está muito satisfeito.

A escala SATIS-BR faz parte de um questionário que possui um total de 44 questões, além das quantitativas da própria escala (12 itens), há questões descritivas e qualitativas referentes à percepção dos usuários sobre alguns aspectos dos serviços recebidos. As questões qualitativas ao final da escala buscam avaliar: o que o usuário considera melhor no CAPSad, o que considera pior e o que deveria ser melhorado.

O projeto foi submetido no Comitê de Ética em Pesquisa da Escola de Enfermagem da Universidade de São Paulo, seguindo as determinações da Resolução 196/96, do Conselho Nacional de Ética em Pesquisa - CONEP/ CNS e aprovado com o no 1001/2011.

Os procedimentos estatísticos foram processados por meio do programa SPSS, versão 22.0, envolvendo a análise descritiva dos dados, testes inferenciais para a análise da correlação dos escores dos fatores da escala entre si (índice de correlação de Spearman) e construção de intervalos de confiança para cada uma das variáveis quantitativas.

\section{RESULTADOS}

\section{Descrição da Amostra}

O perfil dos participantes está descrito na distribuição de frequências dos dados (Tabela 1) segundo gênero, etnia, faixa etária, estado civil, escolaridade, fonte de renda e renda familiar no último mês.

Tabela 1 -Distribuição das Frequências de Respostas. Botucatu, SP, Brasil, 2013.

\begin{tabular}{|c|c|c|}
\hline Variáveis & Freq. Absoluta & Freq. percentual \\
\hline \multicolumn{3}{|l|}{ Gênero } \\
\hline Feminino & 8 & $32 \%$ \\
\hline Masculino & 17 & $68 \%$ \\
\hline \multicolumn{3}{|l|}{ Etnia } \\
\hline Branca & 22 & $88 \%$ \\
\hline Negra & 3 & $12 \%$ \\
\hline \multicolumn{3}{|l|}{ Faixa etária } \\
\hline de 26 a 30 & 4 & $16 \%$ \\
\hline de 31 a 40 & 9 & $36 \%$ \\
\hline de 41 a 50 & 5 & $20 \%$ \\
\hline de 51 a 69 & 7 & $28 \%$ \\
\hline \multicolumn{3}{|l|}{ Estado Civil } \\
\hline Casado/com companheiro & 9 & $36 \%$ \\
\hline Divorciado/Separado & 2 & $8 \%$ \\
\hline Viúvo & 2 & $8 \%$ \\
\hline Solteiro & 12 & $48 \%$ \\
\hline \multicolumn{3}{|l|}{ Escolaridade } \\
\hline Ensino Fundamental Incompleto & 10 & $40 \%$ \\
\hline Ensino Fundamental Completo & 3 & $12 \%$ \\
\hline Ensino Médio Incompleto & 2 & $8 \%$ \\
\hline Ensino Médio Completo & 8 & $32 \%$ \\
\hline Curso Técnico Completo & 1 & $4 \%$ \\
\hline Ensino Superior Incompleto & 1 & $4 \%$ \\
\hline \multicolumn{3}{|l|}{ Fonte de Renda } \\
\hline Emprego & 9 & $36 \%$ \\
\hline Aposentadoria & 3 & $12 \%$ \\
\hline Auxílio-doença & 5 & $20 \%$ \\
\hline $\begin{array}{l}\text { Benefício de Prestação Continuada } \\
\text { - BPC/LOAS }\end{array}$ & 1 & $4 \%$ \\
\hline Bolsa Família & 1 & $4 \%$ \\
\hline Não Tem Renda & 6 & $24 \%$ \\
\hline \multicolumn{3}{|l|}{ Renda Familiar último mês } \\
\hline Até 2 salários mínimos & 14 & $56 \%$ \\
\hline Entre 2 a 4 salários mínimos & 9 & $36 \%$ \\
\hline Entre 4 a 10 salários mínimos & 2 & $8 \%$ \\
\hline Total & 25 & $100 \%$ \\
\hline
\end{tabular}

Em sua maioria, os pacientes eram do sexo masculino (68\%), solteiros (48\%), com escolaridade predominante de ensino fundamental incompleto (40\%). A idade média era de 43 anos (dp 12,42), sendo a mínima de 26 e a máxima de 69 anos. Parte expressiva dos pacientes possuía renda própria (76\%), sendo as principais fontes de renda o emprego (36\%) e o auxílio-doença (20\%). A renda familiar no último mês foi de até dois salários mínimos (56\%). 
No que concerne à situação de moradia, os usuários vivem: com familiar (48\%), especialmente mãe ou pai, com cônjuge/companheiro(a) (32\%), sozinhos (8\%), com amigo fora do CAPSad (4\%), com cuidador (4\%) e em albergue municipal (4\%). Em referência a ser ou não a primeira admissão do usuário no serviço, 60\% afirmaram que foi a sua primeira admissão nesse CAPSad. No que diz respeito ao tempo em que os usuários são assistidos neste serviço, $24 \%$ frequentam este CAPSad entre sete a doze meses, $48 \%$ entre um a dois anos e $28 \%$, a mais de dois anos.

A idade do usuário quando percebeu o uso abusivo/dependência da substância foi em média de 29 anos (dp $11,9)$ e uma mediana de 26 anos, tendo na faixa de 14 a 30 anos um percentual de $68 \%$.

Em relação à internação psiquiátrica antes de iniciar o tratamento no CAPSad, $68 \%$ sinalizaram que foram internados, sendo $56 \%$ em hospital psiquiátrico e $22 \%$ em comunidades terapêuticas.

Já em relação à internação psiquiátrica durante o tratamento no CAPSad, 36\% sinalizaram que tiveram internações em hospital psiquiátrico $(28 \%)$ e em comunidades terapêuticas (8\%).

Quanto ao uso de psicofármacos, 96\% referem que em nenhuma situação precisou comprar a medicação que faz uso. Cerca de $80 \%$ referem "sempre" seguir a prescrição médica, $4 \%$ seguem "frequentemente" e 16\% seguem "às vezes".

\section{Satisfação dos Pacientes com o Serviço de Saúde Mental}

A tabela 2 apresenta os resultados descritivos dos escores de satisfação global e por fatores $(1,2,3)$ dos usuários para cada item da escala SATIS-BR, em termos das médias e desvios-padrão $(n=25)$.

Tabela 2 -Medidas Descritivas dos Escores da Escala SATIS-BR. Botucatu, SP, Brasil, 2013.

\begin{tabular}{|c|c|c|c|c|}
\hline Item da Escala & Média & $\begin{array}{c}\text { Desvio- } \\
\text { padrão }\end{array}$ & $\begin{array}{c}\text { Escore } \\
\text { Mínimo }\end{array}$ & $\begin{array}{c}\text { Escore } \\
\text { Máximo }\end{array}$ \\
\hline Global do SATIS BR & 4,0 & 0,3 & 3,1 & 4,5 \\
Fator 1: Competência e Compreensão & 4,1 & 0,38 & 2,5 & 4,7 \\
Fator 2: Ajuda recebida e acolhida & 4,3 & 0,37 & 3,3 & 4,3 \\
Fator 3: Condições físicas e conforto & 4,2 & 0,5 & 2,5 & 4,1 \\
\hline
\end{tabular}

Os resultados identificaram uma avaliação positiva em todos os itens, com média em torno de 4,0 (dp 0,3) para o quadro global de satisfação, tendo uma variação de 3,1 a 4,5. As questões referentes à ajuda recebida e acolhida do usuário no CAPSad (fator 2), foram as que receberam melhor avaliação, com uma média de 4,3 (dp 0,37) variando de 3,3 a 4,3.
O item da escala com indicativo mediano de satisfação referiu-se à satisfação do usuário com as condições físicas e conforto do CAPSad (fator 3), ficando com uma média de 4,2 (dp 0,5), variando entre 2,5 a 4,1.

As questões que avaliaram a Competência e a Compreensão da Equipe (fator 1) receberam a pior avaliação, com média de 4,1 (dp 0,38$)$, apresentando a maior variação entre 2,5 a 4,7.

Foram também apresentadas as porcentagens de algumas questões da escala não referentes aos fatores acima citados e considerados importantes na avaliação da satisfação dos usuários.

A questão de participação, também representa uma limitação quando os usuários são questionados acerca de tomar parte no processo de avaliação das atividades desenvolvidas no CAPS e apenas 36\% refere participar. Cerca de $60 \%$ consideram que a equipe do CAPS, em geral ajuda o usuário.

O CAPS atende as necessidades de $80 \%$ dos usuários que se dizem satisfeitos, e $16 \%$ muito satisfeitos. Para $36 \%$ dos usuários, o tipo de ajuda dada pelo CAPS significou alguma ajuda, e muita ajuda, para 64\%. Para $100 \%$ dos usuários, os serviços recebidos no CAPS os ajudaram a lidar mais eficazmente com seus problemas. O acesso ao CAPS é considerado fácil para $72 \%$ dos usuários e ocorrem através de transporte público, $44 \%$, ou por condução própria, 32\%. Quanto ao tempo de deslocamento até o CAPS, 32\% demora entre 31 a 45 minutos.

Quanto às questões qualitativas do instrumento aplicado aos usuários, foram considerados pontos positivos: a dinâmica dos grupos terapêuticos, o ambiente do CAPSad e o relacionamento com os colegas.

A avaliação positiva dos usuários, entretanto, não impede que $40 \%$ dos usuários considerem que o serviço no CAPSad precisa ser melhorado.

Dentre as melhorias solicitadas as mais referidas foram: aumento do número de salas para atendimento; maior número de profissionais; atendimento noturno e alimentação; melhoria da estrutura física do prédio; maior qualificação dos técnicos; material para oficinas terapêuticas e aumento de atendimento psicológico individual.

Os resultados da medida de associação entre os fatores relativos aos graus de satisfação global e seus fatores: competências e compreensão, ajuda e acolhida e condições físicas e de conforto, estão apresentadas na tabela 3 . 
Tabela 3 - Medidas de Associação de Spearman para Avaliação da Satisfação dos Usuários. Botucatu, SP, Brasil, 2013.

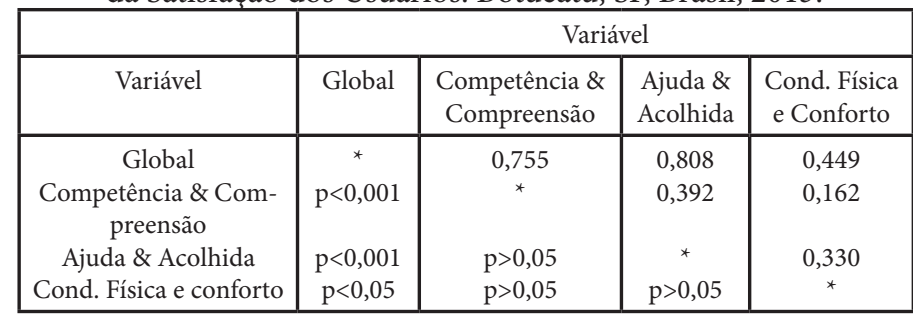

A tabela 3 apresenta as correlações de Spearman dos fatores entre si e com a escala global. Os resultados mostraram associação significativa dos escores dos três fatores com o escore global, e ausência de associação entre os fatores isolados.

Todos os fatores estão associados positivamente $(\mathrm{p}<0,05)$ com o escore global e os resultados mostraram que o escore global eleva-se quando há elevação dos fatores. Como na comparação dois a dois entre os fatores não apresenta significância estatística, não há associação entre eles e seus resultados elevados ou baixos ocorreram independentemente entre os fatores.

\section{DISCUSSÃO}

Dados da pesquisa e estudos apontam predominância da população masculina no tratamento acerca do problema da dependência de drogas, apontando uma baixa frequência das mulheres neste tratamento. Sabe-se, no entanto, que as questões culturais e o preconceito, ainda fazem parte da visão de mundo da maioria dos profissionais de saúde, refletindo-se no decorrer das atividades de atendimento ao usuário de AOD, principalmente em relação à mulher, o que pode ajudar a compreender a significativa diferença numérica no total de usuários (Pelisoli \& Moreira, 2005; Pelisoli \& Moreira, 2009; Marini, 2011; Pelisoli et al, 2011; Batista, Batista \& Constantino, 2012; Pereira et al, 2012).

Estudos afirmam que as mulheres procuram menos os serviços de atendimento a usuários de AOD do que os homens, por razões como o estigma social em relação ao papel da mulher e por conta de o próprio perfil de usuário da mulher ser diferente do homem (Cesar, 2006; Peixoto et al, 2010) Comparando dados da presente pesquisa com outros estudos, notou-se similaridade nas questões referentes à: faixa etária, estado civil e situação ocupacional (Batista et al, 2012; Pelisoli \& Moreira, 2005). A média de idade dos usuários, neste estudo, foi de 43 anos, próximo ao observado na população pesquisada em Lorena (SP) que a idade média era de 41 anos e maior que na população do Rio Grande do
Sul, que apresentou a média de 30,35 anos (Kantorski et al, 2009; Pereira et al, 2012).

Também houve a predominância da baixa escolaridade, a questão de estar fora do mercado de trabalho e a de não ter companheiro foi similar às características da amostra de estudos em CAPSad (Marini, 2011; Batista et al, 2012).

No que se refere ao vínculo empregatício dos usuários, tem-se na presente pesquisa que apenas $36 \%$ estavam empregados no momento em que foram entrevistados. A problemática do desemprego é uma questão de ordem estrutural e conjuntural; entretanto, essa questão torna-se mais significante nos usuários abusivos de AOD, visto que estes representam um segmento marginalizado dentro do conjunto dos socialmente excluídos (Batista, Batista, \& Constantino, 2012).

Tal fato torna sua inserção e permanência no mercado de trabalho ainda mais restrita, em virtude de apresentarem dificuldades em desempenhar satisfatoriamente suas funções, devido aos efeitos e consequências biopsicossociais específicas de cada tipo de uso abusivo de AOD, caso não estejam em abstinência.

Confrontando os dados encontrados com outras pesquisas, verificou-se que os usuários desse estudo apresentam uma melhor adesão do que nos outros estudos, pois $48 \%$ frequentam o serviço a mais de seis meses, enquanto em outros estudos mais de 50\% dos usuários não permanecem em tratamento na instituição (Marini, 2011; Pelisoli et al, 2011). Há um tempo pré-estabelecido para considerar que o usuário aderiu ao tratamento. A partir do terceiro mês do tratamento, pode-se dizer que o usuário aderiu. A boa adesão ocorre se o usuário permaneceu no tratamento por um período mínimo de seis meses e muito boa quando a permanência é superior a seis (Pelisoli et al, 2011). A aderência ou adesão ao tratamento pode ser entendida como o grau em que o paciente segue as recomendações médicas, ou de outro profissional da saúde consultado, e é entendida como o retorno e a manutenção do tratamento indicado e inclui a frequência, até o momento de sua alta (Pelisoli et al, 2011).

Revisão recente acerca de processos avaliativos em serviços de saúde mental aponta que a satisfação do usuário para com a atenção recebida está diretamente ligada aos vínculos forma $\neg$ dos com a equipe de saúde, ao acolhimento realizado nas consultas e ao acompanha-mento durante todo o tratamento. Tais elementos mostraramse positivos em relação ao melhor sucesso e adesão ao tratamento (Oliveira et al, 2014). 
A satisfação dos pacientes vem sendo atrelada a várias questões, entre elas: adesão ao tratamento, características do atendimento, variáveis sociodemográficas e clínicas dos pacientes, redução da sintomatologia, competência da equipe, qualidade do relacionamento profissional $x$ paciente, acessibilidade ao serviço, qualidade das instalações, continuidade dos cuidados e também a informação recebida (Silva et al, 2012; Oliveira et al, 2014).

Quanto à satisfação do usuário (SATIS-BR), os resultados indicaram um bom grau de satisfação com o CAPSad, tanto na escala global, como nos seus fatores. Entretanto, a média global de satisfação nesse estudo foi 4,0 ; sendo menor quando comparada a outros estudos, em que a média foi de 4,52 e de 4,40 (Kantorski et al, 2009; Kantorski et al, 2009b).

Em relação aos fatores, o que obteve a maior pontuação foi o fator 2, que avalia a ajuda recebida e a acolhida da equipe $(4,3)$; corroborando com outra pesquisa realizada em um Centro de Referência em Saúde Mental, em que os usuários apresentaram um grau mais elevado de satisfação com relação a esses itens, comparativamente aos demais aspectos (Camilo et al, 2012).

Ressaltamos que a maior pontuação nesse fator pode ser confirmada por meio de outras questões que demonstraram resultados como: $100 \%$ dos usuários afirmar que o CAPSad trouxe alguma ou muita ajuda, assim como $100 \%$ afirmar que o CAPSad os auxiliou a lidar melhor com seus problemas.

Por outro lado, observou-se menor satisfação com as condições gerais das instalações do CAPSad (fator 3), com média de 4,2; dado confirmado em outro estudo e com os itens referentes à competência e compreensão da equipe, com média de 4,1 (Kantorski et al, 2009b).

Mesmo com uma significativa avaliação positiva dos usuários, nas questões qualitativas foram apontados aspectos que merecem maior investimento e readequação no serviço, destacando-se entre estes: melhora da estrutura física, maior qualificação dos técnicos, ampliação do número de profissionais e do horário de atendimento. Pode-se inferir, portanto, que uma das maiores fragilidades do CAPSad está nas condições de infraestrutura dos serviços, aspecto presente também em outros estudos (Kantorski et al, 2009; Silva et al, 2012).

\section{CONCLUSÃO}

Os resultados deste estudo demonstraram que a avaliação dos usuários sobre o CAPSad se situa entre a satisfação e muita satisfação. A maior satisfação derivou dos itens que avaliaram a ajuda recebida e a acolhida da equipe, demonstrando que apesar das fragilidades e dificuldades o CAPSad cumpre com alguns objetivos a que se propõe como o acolhimento e a criação de vínculos, seja com os técnicos ou com os colegas que também frequentam o CAPSad, colaborando com a ampliação da rede social do usuário. Constatando-se, portanto, a importância deste serviço para o tratamento dessa população.

Entretanto, mesmo com a avaliação positiva dos usuários, foram apontados aspectos que merecem maior investimento: estrutura física, formação e ampliação da equipe de saúde.

Como este trabalho foi realizado em um único serviço de saúde mental, não pode ser generalizado para outros serviços. Deste modo, espera-se que os resultados apresentados orientem a política local na elaboração de novas ações que ampliem a atenção aos usuários de AOD, bem como a sua permanência no tratamento, no sentido de promover a sua reinserção social e a diminuição dos sintomas apresentados.

\section{REFERÊNCIAS}

Amarante, P. (2008). Saúde mental, desinstitucionalização e novas estratégias de cuidado. In L. Giovanella, S. Escorel, L. V. C. Lobato, J. C. Noronha, \& A. I. Carvalho (Orgs.), políticas e sistema de saúde no Brasil (Cap. 20). Editora Fiocruz/CEBES.

Bandeira, M., Pitta, A. M. F., \& Mercier, C. (2000). Escala de avaliação da satisfação dos usuários em serviços de saúde mental: SATIS-BR. Jornal Brasileiro de Psiquiatria, 49(8), 293-300.

Batista, L. S. S., Batista, M., \& Constantino P. (2012). Perfil dos usuários de substâncias psicoativas do CAPSad em 2000 e 2009, Campo dos Goyatacazes, RJ. Perspectivas Online: Biológicas \& Saúde, 7(2), 23-38. 
Camilo, C. A., Bandeira, M., Leal, R. M. A. C., \& Scalon, J. D. (2012). Avaliação da satisfação e sobrecarga em um serviço de saúde mental. Ciência e Saúde Coletiva, 20(1), 82-92.

Cesar, B. A. L. (2006). Alcoolismo feminino: Um estudo de suas peculiaridades. Jornal Brasileiro de Psiquiatria, 55(3) 208-2011.

Furtado J. P. (2001). A avaliação como dispositivo. Tese de Doutorado. Campinas: Programa de Pós-Graduação em Saúde Coletiva da Faculdade de Ciências Médicas/ Universidade Estadual de Campinas.

Kantorski, L. P., Jardim, V. R., Wetzel, C., Olschowsky, A., Schneider, J. C., Resmini, F., Heck, R. M., Bielemann, V. L. M., Schwartz, E., Coimbra, V. C. C., Lange, C., \& Sousa, A. S. (2009). Contribuições do estudo de avaliação dos centros de atenção psicossocial da região sul do Brasil (CD-ROM). Cadernos Brasileiros de Saúde Mental. Acedido em 13 de Janeiro de 2011, em http:// www.abrasme.org.br/cbsm/artigos/artigos/20_Luciane_Kantorski.pdf

Kantorski, L. P., Jardim, V. R., Wetzel, C., Olschowsky, A., Schneider, J. F., \& Heck, R. M. (2009b). Satisfação dos usuários dos centros de atenção psicossocial da região Sul do Brasil. Revista de Saúde Pública, 43(Suppl. 1), 29-35.

Marini, M. (2011). Preditores de adesão ao projeto terapêutico em Centro de Atenção Psicossocial álcool e outras drogas. Dissertação de Mestrado, Universidade Federal do Rio Grande do Sul, Escola de Enfermagem, Programa de Pós-Graduação em Enfermagem, Porto Alegre, Br-Rs.

Ministério da Saúde. Secretaria Executiva. Coordenação Nacional de DST e Aids (2003). A política do Ministério da Saúde para atenção integral a usuários de álcool e outras drogas. Brasília: Ministério da Saúde.
Oliveira, M. A. F., Cestari, T. Y, Pereira, M. O., Pinho, P. H., Gonçalves, R. M. D. A., Claro, H. G. (2014). Processos de avaliação de serviços de saúde mental: uma revisão integrativa. Saúde em Debate, 38(101), 368-378.

Peixoto, C., Prado, C. H. O., Rodrigues, C. P., Cheda, J. N. D., Mota, L. B. T., \& Veras A. B. (2010). Impacto do perfil clínico e sociodemográfico na adesão ao tratamento de pacientes de um Centro de Atenção Psicossocial a Usuários de Álcool e Drogas (CAPSad). Jornal Brasileiro de Psiquiatria, 59(4), 317-321.

Pelisoli, C. da L., \& Moreira A. K. (2005). Caracterização epidemiológica dos usuários do Centro de Atenção Psicossocial Casa Aberta. Revista de Psiquiatria do Rio Grande do Sul, 27(3), 270-277.

Pelisoli, C. da L., \& Moreira, A. K. (2009). O perfil dos usuários do CAPSad Blumenau e as politicas públicas em saúde mental. Psicologia \& Sociedade, 21(3), 324333.

Pelisoli, C. da L., Moreira, A. K. C. F. S., Fé, L. C. M., Moreira, M. A. C, Albuquerque, I. E. M., Silva, M. G., \& Passamani, M. C. (2011). Perfil sociodemográfico e adesão ao tratamento de dependentes de álcool em CAPS-ad do Piauí. Escola Anna Nery, 15(1), 90-95.

Pereira, M. O., Souza, J. M., Costa, A. M., Vargas, D., Oliveira, M. A. F., \& Moura, W. N. (2012). Perfil dos usuários dos serviços de saúde mental do município de Lorena - São Paulo. Acta Paulista de Enfermagem, 25(1), 48-54.

Silva, M. A., Bandeira, M., Scalon, J. D., Quaglia, M. A. C. (2012). Satisfação dos pacientes com os serviços de saúde mental: A percepção de mudanças como preditora. Jornal Brasileiro de Psiquiatria, 61(2), 64-71.

World Health Organization. WHO-SATIS (1996). Consumer's and caregivers' satisfaction with mental health services: A multisite study. WHO Division of Mental Health. 


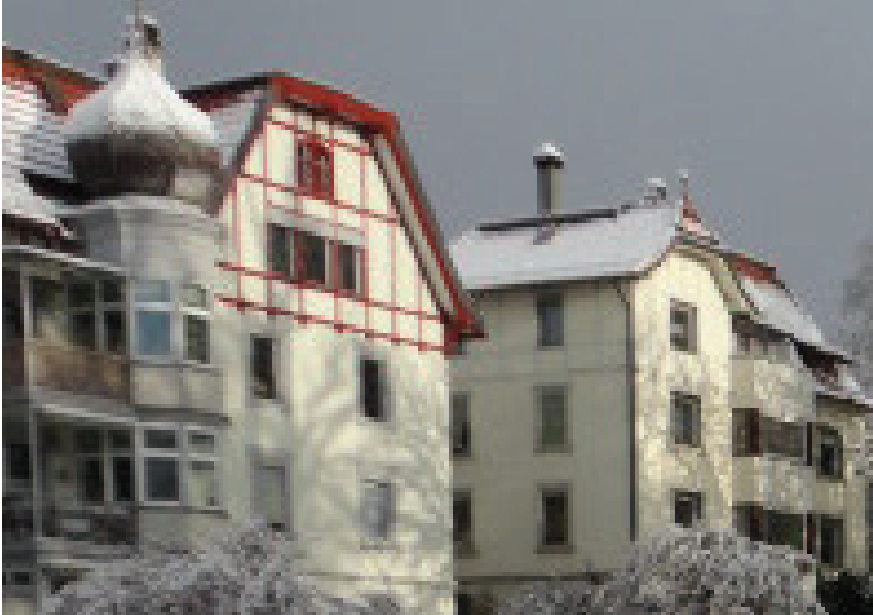

thit

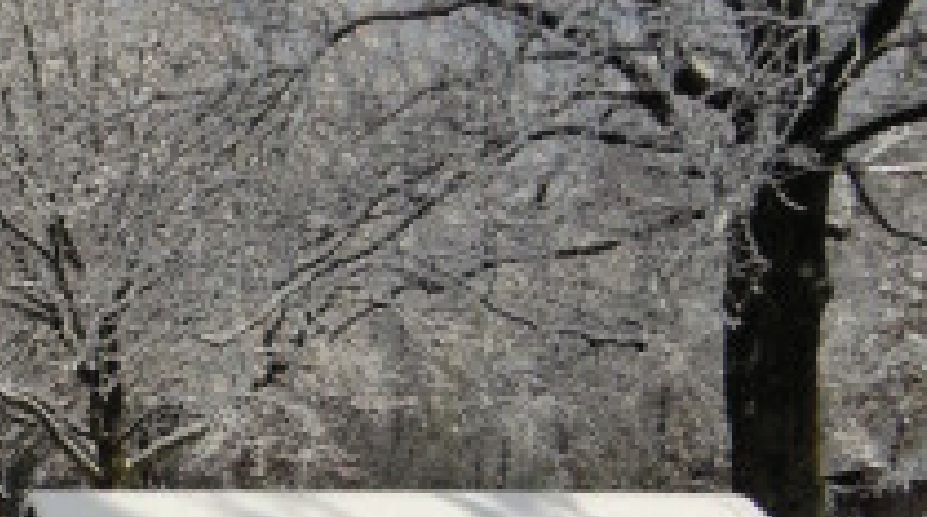

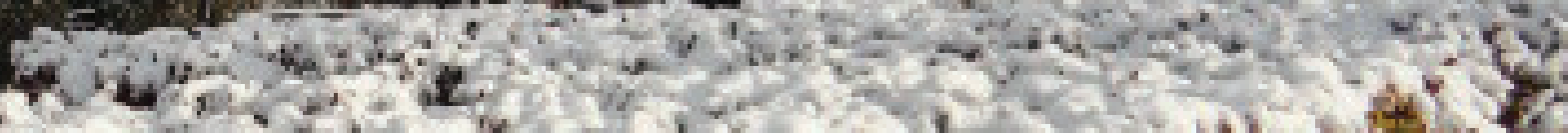
1.5.

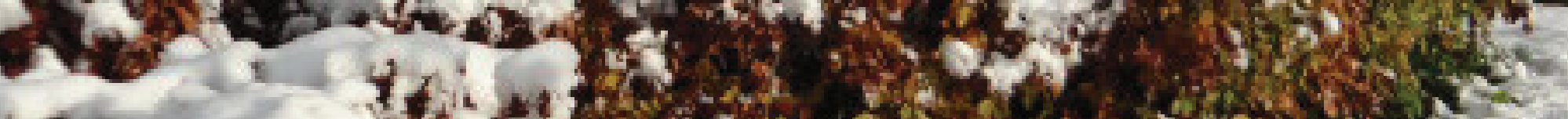

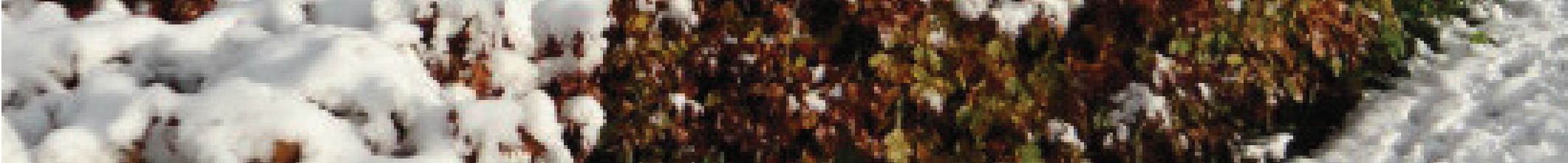

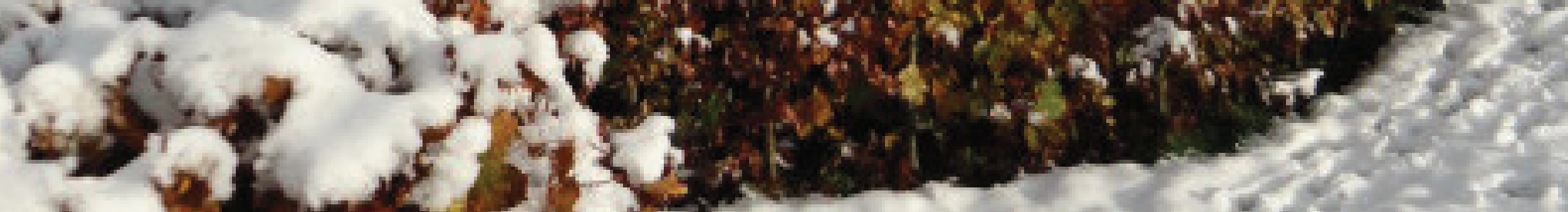
ind 3.ton 045

(i) $0.3 \times, x^{2}$

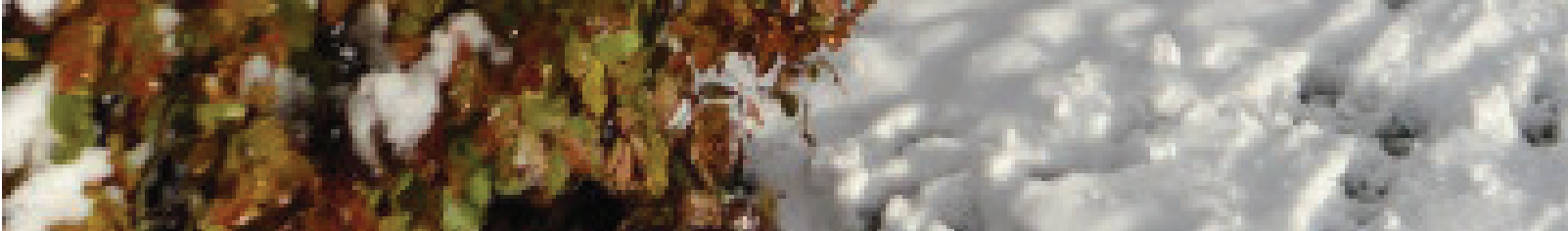

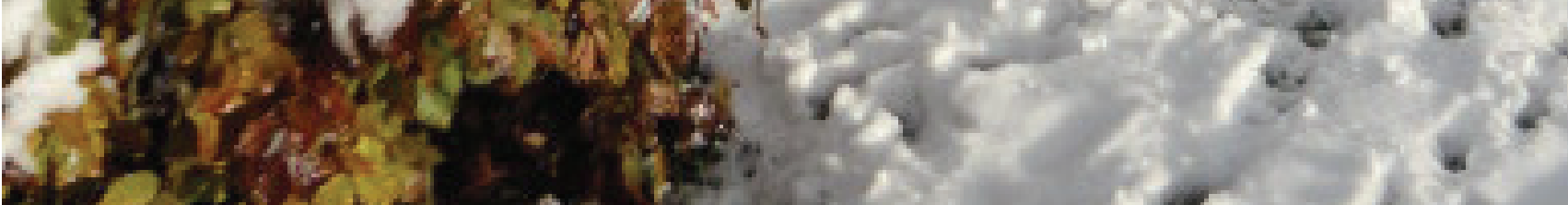
3.

istis. 40 $2^{2}=$ $\ln ^{2}+\mathrm{m}$ 